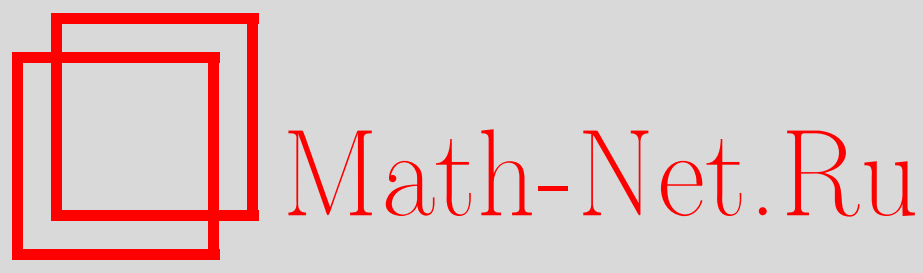

Ю. А. Кашлев, Уравнение баланса и квазитемпература каналированных частиц в состоянии квазиравновесия, TMФ, 2009, том 161, номер 2, 256-266

DOI: https://doi.org/10.4213/tmf6436

Использование Общероссийского математического портала Math-Net.Ru подразумевает, что вы прочитали и согласны с пользовательским соглашением http://www.mathnet.ru/rus/agreement

Параметры загрузки:

IP: 3.82 .47 .9

26 апреля 2023 г., 14:21:11

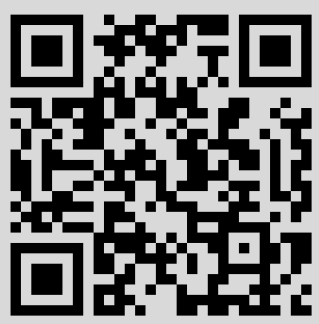




\title{
УРАВНЕНИЕ БАЛАНСА И КВАЗИТЕМПЕРАТУРА КАНАЛИРОВАННЫХ ЧАСТИЦ В СОСТОЯНИИ КВАЗИРАВНОВЕСИЯ
}

\begin{abstract}
Методами неравновесной статистической термодинамики получено уравнение баланса поперечной энергии и поперечного импульса для быстрых атомных частиц, движущихся в режиме плоскостного каналирования. На основе решения этого уравнения получено выражение для поперечной квазитемпературы в состоянии квазиравновесия через основные параметры теории. Показано, что равновесное значение квазитемпературы каналированных частиц устанавливается за счет диффузии частиц в пространстве поперечных энергий ("разогрев" подсистемы), диссипативного процесса ("охлаждение") и ангармонических эффектов колебания частиц между стенками канала (перераспределение энергии по колебательным степеням свободы - внутренняя термализация подсистемы). Согласно оценкам для частиц с энергией порядка 1 МэВ значения квазитемпературы укладываются в температурный интервал, характерный для низкотемпературной плазмы.
\end{abstract}

Ключевые слова: неравновесная статистическая термодинамика, уравнение баланса, каналированная частица, электрон, ядерный спин, квазитемпература, диффузия, диссипация энергии, ангармонизм.

1. Понятие квазитемпературы как модуля равновесного распределения выделенной подсистемы частиц (квазичастиц, спинов) с успехом применяется в случае систем, далеких от полного термодинамического равновесия. Перечислим несколько характерных примеров применения этого понятия:

- квазитемпература при наличии взаимодействия между частицами (квазичастицами, спинами);

- квазитемпература в случае слабого взаимодействия между некоторыми (поступательными, внутренними) степенями свободы, когда соответствующие релаксационные процессы независимы;

- квазитемпература при наличии взаимодействия частиц с термостатом и дополнительного обмена энергией между внутренними степенями свободы подсистемы.

Первый случай характерен для подсистемы ядерных спинов, изолированных от решетки, когда спины за счет спин-спинового взаимодействия переходят в состояние внутреннего квазиравновесия, причем вероятность нахождения подсистемы в

${ }^{*}$ Институт металлургии и материаловедения им. А. А. Байкова РАН, Москва, Россия 
данном энергетическом состоянии описывается больцмановским распределением [1]. Второй случай реализуется в плазме [2], [3], когда модулем распределения по всем степеням свободы, кроме поступательной, является эффективная электронная температура. Точнее, распределение тяжелых частиц (ионов, нейтралов) по поступательным степеням свободы определяется температурой тяжелой компоненты плазмы, тогда как модулем распределения по всем остальным степеням свободы является температура электронов. Что касается третьего случая, то он имеет прямое отношение к "горячим" атомам, движущимся в кристаллических твердых телах, в том числе к высокоэнергетическим каналированным частицам (КЧ) [4], [5]. Эффект плоскостного каналирования можно рассматривать с точки зрения неравновесной статистической термодинамики, поскольку КЧ представляют собой самостоятельную термодинамическую подсистему. В настоящей работе мы будем изучать термодинамику КЧ, прежде всего поперечную квазитемпературу этой подсистемы, ограничиваясь учетом электронных столкновений.

В статье используется система единиц, в которой постоянная Больцмана и постоянная Планка равны единице.

2. Если рассматривать движение КЧ на больших глубинах проникновения частиц в кристалл, $L>L_{\text {coh }}\left(L_{\text {coh }}\right.$ - длина когерентности [4]), то детали их начального распределения становятся несущественными, так что число переменных, необходимых для описания системы, сокращается. Исключая для простоты хаотическую часть пучка, разделим полную систему на две слабо взаимодействующие подсистемы: подсистему $\{1\}$ - термостат, включающий идеальную решетку и электронный газ, и подсистему $\{2\}$ - быстрые атомные частицы, движущиеся в плоскостном канале [6]. Пусть переменная $P_{1 i}=H_{i}$ - это гамильтониан $i$-й подсистемы, $P_{2 i}$ и $P_{3 i}-$ полный импульс $\mathcal{P}_{i}$ и полное число частиц $N_{i}$ соответственно, $i=1,2$. Как уже упоминалось, мы ограничиваемся учетом рассеяния КЧ на электронах, поскольку эти столкновения являются наиболее частыми [6]. Полный гамильтониан системы запишем в форме

$$
H=H_{1}+H_{2}, \quad H_{1}=H_{\mathrm{e}}, \quad H_{2}=H_{0}^{(2)}+H_{\mathrm{int}}^{(2)}+\widetilde{H}_{\mathrm{int}}^{(2)},
$$

где $H_{\mathrm{e}}$ - гамильтониан свободных электронов, $H_{0}^{(2)}$ - гамильтониан КЧ, который включает потенциал плоскостного канала (стенки), $H_{\mathrm{int}}^{(2)}=W_{\mathrm{c}}^{(2)}$ - оператор, соответствующий близким столкновениям КЧ с электронами, $\widetilde{H}_{\text {int }}^{(2)}$ - оператор взаимодействия КЧ с плазмонами.

В соответствии с определениями неравновесной статистической термодинамики [7] операторы энтропии и производства энтропии мы представим в виде

$$
\begin{aligned}
& S(t, 0)=\Phi(t)+\sum_{m, i} P_{m i} F_{i m}(t), \quad \Phi(t)=\ln \operatorname{Sp} \exp \left\{\sum_{m, i} P_{m i} F_{i m}(t)\right\} \\
& \dot{S}(t, 0)=\sum_{m, i}\left\{\dot{P}_{m i} F_{i m}(t)+\left(P_{m i}-\left\langle P_{m i}\right\rangle_{l}^{t}\right) \dot{F}_{i m}(t)\right\}
\end{aligned}
$$

где $\Phi(t)$ - функционал Масье-Планка, а неоператорные величины $F_{i m}$ имеют смысл термодинамических параметров, сопряженных средним значениям переменных $P_{m i}$, 
в частности $1 / F_{21}$ - квазитемпература КЧ, $\langle A\rangle_{l}^{t}=\operatorname{Sp}\left(\rho_{l} A\right)$, где $\rho_{l}=e^{-S(t, 0)}$ - квазиравновесный статистический оператор, соответствующий экстремуму информационной энтропии [7].

Представим неравновесный статистический оператор с точностью до членов второго порядка по потенциалу взаимодействия:

$$
\begin{aligned}
\rho(t)=\rho_{l}(t) & +\int_{-\infty}^{0} d t^{\prime} e^{\gamma t^{\prime}} \int_{0}^{1} d \tau e^{-\tau S_{0}(t, 0)} \Delta\left\{\left[F_{21}\left(t+t^{\prime}\right)-\beta_{1}\right] \dot{H}_{2}\left(t^{\prime}\right)\right\} e^{(\tau-1) S_{0}(t, 0)}- \\
& -\int_{0}^{1} d \tau e^{-\tau S_{0}(t, 0)}\left\{\beta_{1} H_{\mathrm{int}}^{(2)}\right\} e^{(\tau-1) S_{0}(t, 0)}
\end{aligned}
$$

Здесь

$$
\begin{gathered}
S_{0}(t, 0)=\Phi_{0}(t)+F_{21}(t)\left\{H_{2}-\mu_{2} N_{2}\right\}+\widetilde{B}, \\
\Phi_{0}(t)=\ln \operatorname{Sp} e^{-F_{21}(t)\left(H_{2}-\mu_{2} N_{2}\right)-\widetilde{B}}, \\
\widetilde{B}=\beta_{1}\left(H_{1}-\mu_{1} N_{1}\right), \quad \Delta A=A-\langle A\rangle^{t}, \quad \gamma \rightarrow+0 .
\end{gathered}
$$

Параметры $F_{i m}(t)$, вошедшие в эти формулы, выбираются так, чтобы истинные средние $\left\langle P_{m i}\right\rangle^{t}=\operatorname{Sp}\left\{\rho(t) P_{m i}\right\}$ были равны квазиравновесным средним $\left\langle P_{m i}\right\rangle_{l}^{t}=$ $\mathrm{Sp}\left\{\rho_{l}(t) P_{m i}\right\}$. Тогда квазиравновесное распределение максимально приближается к истинному неравновесному. Мы используем следующие обозначения: $1 / F_{11}=1 / \beta_{1}-$ температура термостата, $F_{12}=-\beta_{1} \mu_{1}, \mu_{1}$ - химический потенциал термостата, $F_{22}(t)=-F_{21}(t) \mu_{2}, \mu_{2}$ - химический потенциал подсистемы $\{2\}, \lambda=e^{-F_{22}}-$ абсолютная активность КЧ.

Предположим, что первоначально быстрые частицы движутся вдоль кристаллографических плоскостей в направлении оси $z$ с массовой скоростью $V$. При этом они осциллируют между плоскостями, образующими стенки канала, в $x$-направлении. Чтобы исключить направленное движение, перейдем в сопровождающую систему координат. Тогда уравнения движения для операторов $P_{m i}$ запишутся в виде

$$
\begin{gathered}
\dot{H}_{2}=\dot{H}_{2(\mathrm{c})}+n_{2} \sum_{\alpha} f_{\alpha}^{p} V_{\alpha}, \quad \dot{H}_{2(\mathrm{c})}=-i\left[H_{0}^{(2)}, W_{\mathrm{c}}^{(2)}\right], \\
\dot{\mathcal{P}}_{2}^{\alpha}=\dot{\mathcal{P}}_{2(\mathrm{c})}^{\alpha}+n_{2} f_{\alpha}^{p}, \quad \dot{\mathcal{P}}_{1}=0, \quad \dot{N}_{1}=0,
\end{gathered}
$$

где $n_{2}$ - безразмерная плотность КЧ. Индекс "с" в уравнениях (2) указывает на то, что оператор содержит вклад близких столкновений. Что касается дальних столкновений КЧ, то торможение, обусловленное плазмонами, описывается с помощью нелокальной силы

$$
f_{\alpha}^{p}=\frac{2 e^{2}}{\pi^{2}} \int d \omega \int d \mathbf{q} \frac{1}{q^{2}} q^{\alpha} \operatorname{Im} \mathcal{E}^{-1}(\mathbf{q}, \omega) \cdot \frac{1}{2} \delta\left(\omega-(\mathbf{q} \mathbf{V})-\frac{q^{2}}{2 m}\right),
$$

где $\mathcal{E}(\mathbf{q}, \omega)$ - диэлектрическая функция электронов, $\alpha=(x, y, z)$.

Если считать, что обмен энергией между подсистемой $\{2\}$ и термостатом является слабым, то достаточно ограничиться стационарным вариантом теории [7]. В этом случае, усредняя операторные уравнения (2) с неравновесным оператором (1a) 
в условиях стационарности термодинамических параметров, получим уравнение баланса поперечной энергии

$$
\begin{aligned}
n_{2} \sum_{\alpha} f_{\alpha}^{p} V_{\alpha} & +\int_{-\infty}^{0} d t^{\prime} e^{\gamma t^{\prime}}\left(\dot{H}_{2(\mathrm{c})}, \dot{H}_{2(\mathrm{c})}\left(t^{\prime}\right)\right)\left(F_{21}-\beta_{1}\right)- \\
& -\sum_{\alpha} F_{21} V_{\alpha} \int_{-\infty}^{0} d t^{\prime} e^{\gamma t^{\prime}}\left(\dot{H}_{2(\mathrm{c})}, \dot{\mathcal{P}}_{2(\mathrm{c})}\left(t^{\prime}\right)\right)=0
\end{aligned}
$$

и уравнение баланса поперечного импульса КЧ

$$
\begin{aligned}
n_{2} f_{\alpha}^{p}+\int_{-\infty}^{0} d t^{\prime} e^{\gamma t^{\prime}}\left(\dot{\mathcal{P}}_{2(\mathrm{c})}^{\alpha}, \dot{H}_{2(\mathrm{c})}\left(t^{\prime}\right)\right)\left(F_{21}-\beta_{1}\right)- \\
\quad-\sum_{\beta} F_{21} V_{\beta} \int_{-\infty}^{0} d t^{\prime} e^{\gamma t^{\prime}}\left(\dot{\mathcal{P}}_{2(\mathrm{c})}^{\alpha}, \dot{\mathcal{P}}_{2(\mathrm{c})}^{\beta}\left(t^{\prime}\right)\right)=0 .
\end{aligned}
$$

Используя эти уравнения, найдем замкнутое уравнение для $F_{21}$. Для этого, во-первых, пренебрежем "недиагональными" корреляционными функциями в (3), поскольку они дают вклад более высокого порядка по потенциалу взаимодействия, чем “диагональные" [8]. Во-вторых, используя (3б), получим выражение для $f^{p}$ через основные функции теории и подставим его в (3a). В результате с точностью до членов второго порядка имеем

$$
n_{2} F_{21} \sum_{\alpha, \beta}\left\langle V_{\alpha} V_{\beta}\right\rangle_{0} \mathcal{D}_{p_{\alpha} p_{\beta}}+\left(F_{21}-\beta_{1}\right)\left\langle\left(H_{2}\right)^{2}\right\rangle_{0} \nu_{E_{\perp}}=0 .
$$

Здесь $\langle\ldots\rangle_{0}=\operatorname{Sp}\left(\rho_{0} \ldots\right), \rho_{0}=e^{-S_{0}(0,0)}$

$$
\mathcal{D}_{p_{\alpha} p_{\beta}}=\frac{1}{n_{2}} \int_{-\infty}^{0} d t^{\prime} e^{\gamma t^{\prime}}\left(\dot{\mathcal{P}}_{2(\mathrm{c})}^{\alpha}, \dot{\mathcal{P}}_{2(\mathrm{c})}^{\beta}\left(t^{\prime}\right)\right)
$$

- диффузионный коэффициент,

$$
\nu_{E_{\perp}}=\frac{1}{\operatorname{Sp}\left(\rho_{0}\left(H_{2}\right)^{2}\right)} \int_{-\infty}^{0} d t^{\prime} e^{\gamma t^{\prime}}\left(\dot{H}_{2(\mathrm{c})}, \dot{H}_{2(\mathrm{c})}\left(t^{\prime}\right)\right)
$$

- частота релаксации поперечной энергии КЧ.

В сопровождающей системе координат параметр $1 / F_{21}$, вошедший в уравнение баланса энергии-импульса (4), сопряжен средней поперечной энергии КЧ, поэтому его следует интерпретировать как поперечную квазитемпературу КЧ в состоянии квазиравновесия. Поскольку поперечные колебания КЧ совершаются только в $x$-направлении, коэффициент (5) преобразуется в функцию $\mathcal{D}\left(p_{x}\right)$, зависящую от одной переменной. Учтем, что воздействие КЧ на электроны подобно воздействию пробного заряда, частота возбуждения которого (при переходе КЧ из основного состояния в $n$-е возбужденное) равна $\omega_{n 0}$. Тогда диффузионную функцию можно представить в обычном виде:

$$
\mathcal{D}\left(p_{x}\right)=\frac{1}{(2 \pi)^{3}} \Omega \sum_{n} \int d \mathbf{k} \widetilde{w}_{1}\left(\mathbf{k}, \omega_{n 0}\right) k_{x}^{2} .
$$


Здесь

$$
\widetilde{w}_{1}\left(\mathbf{k}, \omega_{n 0}\right)=-\frac{1}{2}(4 \pi)^{3}\left|U_{1}(k)\right|^{2} \operatorname{Im}\left\langle\left\langle\rho_{\mathbf{k}} \mid \rho_{\mathbf{k}}^{\dagger}\right\rangle\right\rangle_{\omega_{n 0}}
$$

- вероятность перехода из основного состояния КЧ $(s=0)$ в возбужденное $(s=n)$, $\left\langle\left\langle\rho_{\mathbf{k}} \mid \rho_{\mathbf{k}}^{\dagger}\right\rangle\right\rangle_{\omega_{n 0}}$ - фурье-компонента двухвременной функции Грина [7], $\rho_{\mathbf{k}}-$ фурье-компонента электронной плотности, $U_{1}(k)=Z_{1} e^{2} / k^{2}, Z_{1} e-$ заряд КЧ, $\Omega$ - единичный объем (в атомных единицах).

3. Скорость изменения энергии $E_{\perp}$ при наличии всех возможных переходов из основного состояния в возбужденное равна

$$
\sum_{n}\left(E_{\perp n}-E_{\perp 0}\right) w_{0 n}=\operatorname{Re}\left\{i\left\langle\left[H_{1}, W_{\mathrm{c}}^{(2)}\right]\right\rangle_{0}\right\}
$$

где $E_{\perp n}-E_{\perp 0}=\Delta E_{n 0}-$ энергия, переданная электронам, $w_{0 n}$ - вероятность перехода в единицу времени, сопровождаемого передачей энергии $\Delta E_{n 0}$. Если перейти в систему координат, фиксированную относительно колеблющейся КЧ, то в этой системе рассеивающий центр неподвижен и оператор взаимодействия $\widetilde{W}_{\mathrm{c}}^{(2)}$ имеет тот же вид, что и в отсутствие поперечного движения частицы. Поэтому, совершая унитарное преобразование координат, можно записать

$$
e^{i G} W_{\mathrm{c}}^{(2)} e^{-i G}=\widetilde{W}_{\mathrm{c}}^{(2)}
$$

где $G=-(\Omega)^{1 / 2} m_{\mathrm{e}} v_{-k} u_{x}, m_{\mathrm{e}}$ - масса электрона, $v_{k}$ - фурье-компонента скорости электрона, $u_{x}$ - поперечное смещение КЧ. Если не переходить в подвижную систему и в разложении $W_{\mathrm{c}}^{(2)}$ по $u_{x}$ ограничиться линейным членом, то это приводит к появлению в теории дополнительного условия, налагаемого на смещение [9], а именно $u_{x} \ll a_{\mathrm{TF}}$ (здесь $a_{\mathrm{TF}}-$ радиус экранирования Томаса-Ферми.) В реальных условиях каналирования атомных частиц в кристаллах это условие не выполняется, и преобразование координат (9) позволяет выйти за рамки указанного ограничения. Координаты в выражении (8) также преобразуются с помощью унитарного преобразования под знаком следа. В результате получаем

$$
\sum_{n}\left(E_{\perp n}-E_{\perp 0}\right) w_{0 n}=\operatorname{Re}\left\{\left\langle\left[\left[W_{\mathrm{c}}^{(2)}, G\right], H_{1}\right]\right\rangle_{G}+i\left\langle\left[\left[\left[W_{\mathrm{c}}^{(2)}, G\right], H_{1}\right], G\right]\right\rangle_{0}\right\}
$$

где $\langle\ldots\rangle_{G}=\left(\delta \rho_{G} \ldots\right), \rho_{G}=e^{-i G} \rho_{0} e^{i G}$.

Если имеется только одна частота возбуждения электронов и эта частота равна так называемой естественной частоте $\tilde{\omega}_{n 0}=(\mathbf{k} \mathbf{V})+k^{2} / 2 m$, то в сумме в левой части (10) сохраняется только один член. В этом случае вероятность перехода в условиях обмена энергией между подсистемами $\{1\}$ и $\{2\}$ вычисляется достаточно точно. Действительно, принимая во внимание, что переданная энергия осциллятора равна

$$
\Delta E_{n 0}=\frac{1}{2} m \widetilde{\omega}_{n 0}^{2} u_{x}^{2}
$$

а также используя распределение $\rho_{G}$, после ряда преобразований находим

$$
w_{0 n} \equiv w_{3}\left(\mathbf{k}, \widetilde{\omega}_{n 0}\right)=-2 \pi\left(m \widetilde{\omega}_{n 0}\right)^{-1} \operatorname{Im}\left\langle\left\langle f_{\mathbf{k}} \mid f_{\mathbf{k}}^{\dagger}\right\rangle\right\rangle_{\tilde{\omega}_{n 0}},
$$


где $f_{\mathbf{k}}=-i\left[m_{\mathrm{e}} v_{\mathbf{k}}, W_{\mathbf{c}}^{(2)}\right]$ - фурье-компонента флуктуационной составляющей силы, действующей на электроны со стороны КЧ. Эффект увлечения электронов колеблющимися рассеивающими центрами, которыми являются КЧ, выражается через корреляцию сил, действующих на электроны, т. е. функцию Грина сила-сила (11). Суммируя вероятности перехода с учетом закона сохранения энергии, находим частоты релаксации поперечного импульса $p_{\perp}$ и поперечной энергии $E_{\perp}$ :

$$
\begin{aligned}
& \nu_{p_{\perp}}=-8 \pi \Omega \int_{0}^{\infty} d \omega \int d \mathbf{k}\left|U_{1}(k)\right|^{2} \operatorname{Im}\left\langle\left\langle\rho_{\mathbf{k}} \mid \rho_{\mathbf{k}}^{\dagger}\right\rangle\right\rangle_{\omega} \delta\left(\omega-(\mathbf{k V})-\frac{k^{2}}{2 m}\right), \\
& \nu_{E_{\perp}}=-8 \pi \Omega \int_{0}^{\infty} d \omega \int d \mathbf{k} \frac{1}{m \omega} \operatorname{Im}\left\langle\left\langle f_{\mathbf{k}} \mid f_{\mathbf{k}}^{\dagger}\right\rangle\right\rangle_{\omega} \delta\left(\omega-(\mathbf{k} \mathbf{V})-\frac{k^{2}}{2 m}\right) .
\end{aligned}
$$

Используя уравнения (4), (7), (8), (11) и (12), находим окончательное уравнение баланса поперечной энергии и поперечного импульса:

$$
\begin{aligned}
& \frac{1}{2} \overline{E_{\perp}} 4 n_{2} F_{21} \int_{0}^{\infty} d \omega \int d \mathbf{k} k_{x}^{2}\left|U_{1}(k)\right|^{2} \operatorname{Im}\left\langle\left\langle\rho_{\mathbf{k}} \mid \rho_{\mathbf{k}}^{\dagger}\right\rangle\right\rangle_{\omega} \delta\left(\omega-(\mathbf{k V})-\frac{k^{2}}{2 m}\right)+ \\
& +\overline{E_{\perp}^{2}}(4 \pi)^{-1}\left(F_{21}-\beta_{1}\right) \int_{0}^{\infty} d \omega \int d \mathbf{k} \omega^{-1} \operatorname{Im}\left\langle\left\langle f_{\mathbf{k}} \mid f_{\mathbf{k}}^{\dagger}\right\rangle\right\rangle_{\omega} \delta\left(\omega-(\mathbf{k} \mathbf{V})-\frac{k^{2}}{2 m}\right)=0
\end{aligned}
$$

где

$$
\overline{E_{\perp}}=\operatorname{Sp}\left\{\rho_{0}(0,0) H_{0}^{(2)}\right\}, \quad \overline{E_{\perp}^{2}}=\operatorname{Sp}\left\{\rho_{0}(0,0)\left(H_{0}^{(2)}\right)^{2}\right\} .
$$

Все известные автору статистические теории каналирования [6], [10]-[12] построены в приближении равномерной заселенности уровней поперечной энергии КЧ. В том же приближении можно вычислить средние значения $\bar{E}_{\perp}$ и $\overline{E_{\perp}^{2}}$. В частности,

$$
\bar{E}_{\perp}=\lim _{1 / F_{21} \rightarrow \infty} \operatorname{Sp}\left\{\rho_{0}(0,0) H_{0}^{(2)}\right\}=\lambda \int d E_{\perp} E_{\perp} g\left(E_{\perp}\right),
$$

где $g\left(E_{\perp}\right)$ - плотность числа состояний КЧ [4].

Выполним интегрирование по угловым переменным вектора $\mathbf{k}$ в (13). Тогда решение уравнения баланса относительно $1 / F_{21}$ запишется в виде

$$
\frac{1}{F_{21}}=\frac{1}{\beta_{1}}\left(1+\frac{A}{B}\right)
$$

где

$$
\begin{aligned}
A= & 2 \pi \bar{E}_{\perp} \int d \sigma(k) k \int_{0}^{k v_{0}} d \omega\left\{1-\left(\frac{\omega}{k v_{0}}\right)^{2}\right\} \times \\
& \times N(0) m_{\mathrm{e}} \frac{1}{k_{\mathrm{F}}} \int_{0}^{\infty} d \varepsilon\left\{n_{\mathrm{e}}(\varepsilon+\omega)-n_{\mathrm{e}}(\varepsilon)\right\}, \\
B= & \frac{1}{2(2 \pi)^{3}} \overline{E_{\perp}^{2}} \int_{0}^{\infty} d \omega \frac{1}{\omega} N(0) m_{\mathrm{e}} \frac{1}{k_{\mathrm{F}}} \int_{0}^{\infty} d \varepsilon\left\{n_{\mathrm{e}}(\varepsilon+\omega)-n_{\mathrm{e}}(\varepsilon)\right\} \times \\
& \times \int d \mathbf{k}^{\prime} \int d \mathbf{k}^{\prime \prime}\left|U_{1}\left(k^{\prime \prime}\right)\right|^{2}\left(k^{\prime}\right)^{-2} \frac{\left(\mathbf{k}^{\prime} \cdot \mathbf{k}^{\prime \prime}\right)^{2}}{\left|\mathbf{k}^{\prime}+\mathbf{k}^{\prime \prime}\right|} \delta\left(\left(\mathbf{k}^{\prime} \mathbf{V}\right)+\frac{\left(k^{\prime}\right)^{2}}{2 m}-\omega\right) .
\end{aligned}
$$


Здесь $n_{1}$ - плотность электронов, $n_{\mathrm{e}}(\varepsilon)$ - распределение Ферми,

$$
d \sigma(k)=\left(8 \pi \frac{Z_{1}^{2} e^{4}}{v_{0}}\right) n_{1} \frac{d k}{k^{3}}
$$

- сечение рассеяния, $N(0)$ - плотность состояний электронов на поверхности Ферми, $k_{\mathrm{F}}$ - импульс Ферми. Если электроны рассматривать как вырожденный газ при нулевой температуре, то можно воспользоваться известной [13] диаграммой ион-электронного столкновения в импульсном пространстве. Согласно этой диаграмме величина $k^{\prime}$ удовлетворяет следующим условиям:

$$
\begin{array}{lcl}
k_{\mathrm{F}}-v_{0} m_{\mathrm{e}}<k^{\prime}<k_{\mathrm{F}}+v_{0} m_{\mathrm{e}} & \text { при } & v_{0}<v_{\mathrm{F}}=\frac{k_{\mathrm{F}}}{m_{\mathrm{e}}}, \\
m_{\mathrm{e}} v_{0}-k_{\mathrm{F}}<k^{\prime}<m_{\mathrm{e}} v_{0}+k_{\mathrm{F}} & \text { при } & v_{0}>v_{\mathrm{F}}, \quad v_{0}=V_{z} .
\end{array}
$$

Кроме того, при вычислении $A$ и $B$ для (14) следует учитывать принцип Паули, который сводится к исключению запрещенной сферы на диаграмме столкновений [13].

4. Ангармонизм колебаний КЧ между стенками плоскостного канала оказывает существенное влияние на временну́ю эволюцию пространственного и углового распределений частиц направленного пучка. Разумеется, этим роль ангармонизма в ориентационных явлениях не исчерпывается. В частности, при выводе квазитемпературы КЧ важно учесть не только диффузию частиц в пространстве поперечных энергий и диссипацию энергии КЧ, но и обмен энергией между частицами, который приводит к перераспределению энергии по колебательным степеням свободы подсистемы $\{2\}$. Перераспределение энергии и установление квазиравновесия КЧ обусловлено ангармоническими эффектами четвертого порядка [14]. Известно, что ангармонические поправки к гармоническому гамильтониану рассматриваются как возмущение, которое вызывает переходы частиц (квазичастиц) из одного состояния в другое. В общем случае такие переходы принято связывать с так называемыми "столкновениями", чтобы подчеркнуть аналогию с другими явлениями переноса [14], [15]. Что же касается ангармонизма четвертого порядка, то он приводит к сужению энергетического расстояния между уровнями поперечной энергии частиц (в потенциальной яме, образованной стенками канала) по мере роста номера уровня $s$. Переходы КЧ между неэквидистантными уровнями, важные для процессов передачи энергии, также можно ассоциировать со "столкновениями". Поскольку ангармонический член мал по сравнению с гармоническим, результат "столкновения" вычисляется по теории возмущений. Характерно, что вычисленное таким образом время между "столкновениями" (время жизни колебательной моды) $\tau_{s}$ содержит параметр связи такого же порядка, как и ангармонический член.

Если в качестве модельного потенциала канала использовать негармонический потенциал вида

$$
V(x)=\frac{1}{2} U_{2}\left[1-\cos \left(\frac{\pi}{l} u_{x}\right)\right],
$$


то первый член разложения $\left(\sim u_{x}^{2}\right)$ соответствует гармоническому потенциалу, а второй $\left(\sim u_{x}^{4}\right)$ - поправке на ангармонизм четвертого порядка. Разумеется, эта поправка включает параметр связи четвертого порядка

$$
B_{l m f g}^{\zeta \nu \rho \sigma}=-\frac{\pi}{3 l^{4}} U_{2}\left\langle l\left|u_{x}\right| l+\zeta\right\rangle\left\langle m\left|u_{x}\right| m+\nu\right\rangle\left\langle f\left|u_{x}\right| f+\rho\right\rangle\left\langle g\left|u_{x}\right| g+\sigma\right\rangle .
$$

Здесь переменные $\zeta, \nu, \rho, \sigma$ принимают только два значения \pm 1 . Поэтому обратное время жизни выражается через произведение двух параметров связи четвертого порядка:

$$
\frac{1}{\tau_{s}}=2 \pi\left(\omega_{\mathrm{h}}\right)^{-1} \sum_{\zeta, \nu, \rho, \sigma} w_{s-\Lambda}^{\zeta \nu \rho \sigma} \delta\left(s-s_{0}+\Lambda\right)
$$

где

$$
w_{s}^{\zeta, \nu, \rho, \sigma}=\sum_{\zeta^{\prime}, \nu^{\prime}, \rho^{\prime}, \sigma^{\prime}} B_{s, s+\zeta, s+\zeta+\nu, s+\zeta+\nu+\rho}^{\zeta \nu \rho \sigma} B_{s, s+\zeta^{\prime}, s+\zeta^{\prime}+\nu^{\prime}, s+\zeta^{\prime}+\nu^{\prime}+\rho^{\prime}}^{\zeta^{\prime} \nu^{\prime} \rho^{\prime} \sigma^{\prime} \Lambda^{\prime}}
$$

Кроме того, в (15) использованы следующие обозначения: $U_{2}=\omega_{\mathrm{h}} s_{0}$ - высота потенциального барьера канала, $\omega_{\mathrm{h}}=(\pi / l)\left(U_{2} / m\right)^{1 / 2}$ - частота колебания КЧ в гармоническом приближении, $l$ - полуширина плоскостного канала, $\Lambda=\zeta+\nu+\rho+\sigma$, $\Lambda^{\prime}=\zeta^{\prime}+\nu^{\prime}+\rho^{\prime}+\sigma^{\prime}$. Отметим также, что выражение (15) применимо, если $\omega_{\mathrm{h}} \tau_{s_{0}}>1$.

Анализ показывает (см., например, [1], [16]), что совместное воздействие двух разных факторов приводит к появлению в релаксационных уравнениях дополнительных членов, описывающих динамические эффекты столкновений в условиях интерференции этих воздействий. Например, в теории магнетизма твердых тел [1] имеет место интерференция двух видов взаимодействия: спин-спинового и спин-решеточного. Благодаря этому в выражение для спиновой квазитемпературы вошла перенормированная диссипируемая энергия $\eta_{1}\left(\widetilde{H}_{L}\right)^{2} \Omega$, в которой основной вклад дает сильное (спин-спиновое) взаимодействие, а слабое спин-решеточное - перенормировочный множитель $\eta_{1}$. Соответственно, в задачах теории каналирования имеется интерференция электронного рассеяния и ангармонизма четвертого порядка. Принимая во внимание сходство термодинамических ситуаций при формировании квазитемпературы КЧ и спиновой квазитемпературы, а также полное совпадение структуры выражений для квазитемператур, для введения интерференции в теорию каналирования достаточно воспользоваться той же заменой, что и в теории ядерных спинов (ЯС). А именно, заменить в (14) диссипируемую энергию $B=\overline{E_{\perp}} \nu_{E_{\perp}}$ на перенормированную энергию $B \eta_{2}$. В результате получаем, что

$$
\frac{1}{F_{21}}=\frac{1}{\beta_{1}}\left(1+\frac{1}{B \eta_{2}}\right) \text {. }
$$

Представим явный вид перенормировочного множителя $\eta_{2}$, описывающего вклад ангармонизма четвертого порядка. Транспортное уравнение [16], [17], включающее ангармонические эффекты, является очень громоздким, и, чтобы избежать усложнений, не имеющих непосредственного отношения к основной цели этой статьи, мы ограничимся приложением известного результата работ [16], [17] к задаче плоскостного каналирования. С учетом (15) получаем

$$
\eta_{2}=\operatorname{ch}\left\{\left(\omega_{\mathrm{h}} \tau_{s_{0}}\right)^{-1 / 2}\right\}
$$


5. Обсудим полученные результаты. Стационарная поперечная квазитемпература, которая устанавливается на глубине проникновения, превышающей длину когерентности $L_{\mathrm{coh}}$, определяется тремя процессами. Прежде всего, это диффузия в $E_{\perp}$-пространстве, которую с точки зрения стохастической теории можно рассматривать как случайные блуждания КЧ между уровнями поперечной энергии. В процессе диффузии частицы заселяют все более высокие энергетические уровни [6], [10], так что средняя поперечная энергия подсистемы $\{2\}$ возрастает. Наряду с диффузией в подсистеме КЧ протекает диссипативный процесс, обусловленный увлечением электронов подвижными рассеивающими центрами, т. е. частицами, осциллирующими между стенками канала. Разумеется, диссипативный процесс приводит к уменьшению средней поперечной энергии КЧ. К этим двум процессам следует добавить обмен энергией между колебательными степенями свободы в подсистеме $\{2\}$, который, в свою очередь, обусловлен ангармоническими эффектами.

В соответствии с общими положениями физической кинетики твердых тел [18] будем считать, что частоты релаксации поперечного импульса и поперечной энергии (12) удовлетворяют условию $\nu_{p_{\perp}} \gg \nu_{E_{\perp}}$. В этом случае благодаря тому, что энергия КЧ медленно передается термостату, возможен заметный "разогрев" подсистемы $\{2\}$. Действительно, оценки величины $1 / F_{21}(14)$ для $\alpha$-частиц с энергией порядка 1 МэВ, движущихся в плоскостных каналах монокристалла кремния, дают значения квазитемпературы порядка одного или нескольких эВ. Отсюда следует важный вывод: значения $1 / F_{21}$ соответствуют температурному интервалу, характерному для низкотемпературной плазмы [3].

В заключение отметим еще одно обстоятельство. С точки зрения основ статистической механики между КЧ и ядерными спинами в сильных магнитных полях существуют общие черты не только в структуре основного термодинамического параметра - квазитемпературы, но и в форме энергетических распределений, а также в распределении заселенностей. Сравним в качестве примера распределение КЧ (см. рис. "а") с распределением ЯС (см. рис. "б"), изолированных от решетки. Распределение, представленное на рис. "б", реализуется в экспериментах с фторидом лития [1], который имеет большое время спин-решеточной релаксации. Энергетическое распределение ЯС имеет вид

$$
\tilde{p}\left(E_{s}\right)=\widetilde{G}\left(E_{s}\right) \tilde{n}\left(E_{s}\right) .
$$

Здесь вероятность нахождения спина с энергией $E_{s}$ задается каноническим распределением $\tilde{n}\left(E_{s}\right) \sim \exp \left(-F_{s} E_{s}\right)$ и плотностью состояний $\widetilde{G}\left(E_{s}\right)$. Кривая $\tilde{p}\left(E_{s}\right)$ на рис. "б" иллюстрирует случай, когда быстро меняющаяся функция плотности состояний $G\left(E_{s}\right)$ [19], умноженная на спадающую функцию $\tilde{n}\left(E_{s}\right)$, дает резкий максимум в полном распределении ЯС $\tilde{p}\left(E_{s}\right)$.

В свою очередь, распределение $\mathrm{K} Ч ~ p\left(E_{\perp}\right)$ также представляет собой немаксвелловскую функцию, обрезанную, как показано на рис. "а", в области больших значений поперечной энергии, т. е. при $E_{\perp}=\omega_{s_{0}}=\omega_{\mathrm{h}} s_{0}[20]$. Из сравнения $p\left(E_{\perp}\right)$ и $\tilde{p}\left(E_{s}\right)$ ясно, что энергетические распределения КЧ и ЯС в полной мере совпадают по форме. 


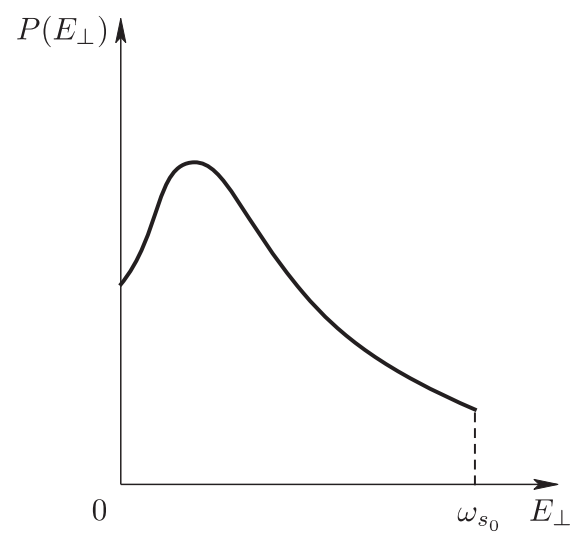

a

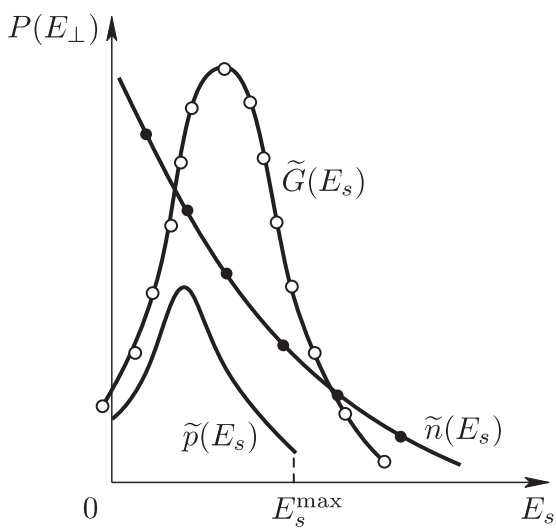

6

Если ограничиться областью положительных квазитемператур, то характер распределения заселенностей КЧ и ЯС один и тот же. А именно, с повышением квазитемпературы заселяются все более и более высокие уровни энергетического спектра системы, причем величины $1 / F_{21}$ и $1 / F_{s}$ определяют ширину распределения заселенностей. В пределе $1 / F_{21} \rightarrow \infty$ и $1 / F_{s} \rightarrow \infty$ достигается равномерная заселенность уровней подсистемы КЧ в модели усеченных осцилляторов и равномерная заселенность уровней спинового газа.

Выше мы неоднократно ссылались на физическую ситуацию, характерную для ЯС, которые находятся под воздействием двух внешних полей: сильного магнитного поля и радиочастотного поля $\widetilde{H}_{1}$. Поэтому для удобства сравнения структуры квазитемпературы КЧ со структурой спиновой квазитемпературы имеет смысл представить явный вид $1 / F_{s}$ в указанном случае. Имеем

$$
\frac{1}{F_{s}}=\frac{1}{\beta_{1}}\left(1+\frac{\widetilde{A}}{\widetilde{B}}\right),
$$

где

$$
\widetilde{A}=\left[\left(\widetilde{H}_{1}\right)^{2}+(\delta h)^{2}\right], \quad \widetilde{B}=\left[\left(\widetilde{H}_{\mathrm{L}}\right)^{2}+\overline{h^{2}}\right],
$$

$(\delta h)^{2}=h^{2}-\overline{h^{2}}$ - квадратичное отклонение от резонанса, $\overline{h^{2}}$ - алгебраическое среднее произведения $h^{(\mathrm{i})} h^{(\mathrm{f})}, h^{(\mathrm{i})}$ - отклонение системы от резонанса в начальный момент, $h^{(\mathrm{f})}$ - отклонение системы от резонанса в конце импульса действия радиочастотного поля, $\widetilde{H}_{\mathrm{L}}$ - квадрат напряженности внутреннего поля (поля Вейса).

\section{Список литературы}

[1] М. Гольдман, Спиновая температура и ЯМР в твердых телах, Мир, М., 1972.

[2] I. P. Shkarofski, T.W. Johnston, M.P. Bachynski, The Particle Kinetics of Plasmas, Addison-Wesley, Reading, MA, 1966.

[3] Л. С. Полак (ред.), Очерки физики и химии низкотемпературной плазмы, Наука, М., 1971.

[4] Ya. A. Kashlev, Phys. Stat. Sol.(b), 190:2 (1995), 379-391.

[5] Ю. А. Кашлев, ТМФ, 126:2 (2001), 311-324. 
[6] J. Lindhard, Mat.-Fys. Medd. Danske Vid. Selsk., 34:14 (1965), 1.

[7] Д.Н. Зубарев, "Современные методы статистической теории неравновесных процессов", Итоги науки и техн. Сер. Соврем. пробл. мат., 15, ВИНИТИ, М., 1980, 131-226.

[8] K. Yamada, Prog. Theoret. Phys., 28:2 (1962), 299-314.

[9] Y. Nagaoka, Prog. Theoret. Phys., 26:5 (1961), 590-610.

[10] Ю. Каган, Ю. В. Кононец, ЖЭТФ, 64:3 (1973), 1042.

[11] J. A. Ellison, S. T. Picraux, Phys. Rev. B, 18:3 (1978), 1028-1038.

[12] M. Rahman, Phys. Rev. B, 52:5 (1995), 3383-3399.

[13] S. P. Ali, D. F. Gallaher, J. Phys. C, 7:14 (1974), 2434-2446.

[14] F. Abel, G. Amsel, M. Braneaux, C. Cohen, A.L'Hoir, Phys. Rev. B, 12:11 (1975), 4617-4627.

[15] Н. Ашкрофт, Н. Мермин, Физика твердого тела, Т. 2, Мир, М., 1979.

[16] Ю. А. Кашлев, ТМФ, 19:3 (1974), 400-413.

[17] Ю.А. Кашлев, Неравновесная статистическая теория скоростей диффузионных процессов в твердых телах (диффузионного деканалирования и диффузионной миграчии), Автореф. дис. ... докт. физ.-мат. наук, Матем. ин-т им. В. А. Стеклова, М., 1982.

[18] Е. М. Лифшиц, Л. П. Питаевский, Теоретическая физика Т. 10. Физическая кинетика, Наука, М., 1979.

[19] Ч. Киттель, Элементарная статистическая физика, ИЛ, М., 1960.

[20] Ю. А. Кашлев, Н. М. Садыков, ТМФ, 116:3 (1998), 442-455.

Поступила в редакцию 16.02.2009, после доработки 29.04.2009 